out a Y chromosome? Biologists have found that gender-straddling and gender-switching behaviours are not at all uncommon in the 'natural' world, either for humans or non-human animals (see page 678). True, modern biotechnology has considerably raised the stakes, and is allowing humans to manipulate their biological make-up to an ever-increasing degree. But it hasn't fundamentally changed the game. And its applications, however unsettling they may be to some people, are not, by definition, 'unnatural'.

This same question of 'natural' versus 'unnatural' also emerges this week in a very different context: an online poll that Nature started in January on the use of neuroenhancing drugs (see page 674). Respondents were asked to report on their non-medical use of drugs such as modafinil and methylphenidate to improve their concentration. These drugs can have mild effects, not all that different from caffeine (a natural substance) or other stimulants. But somehow the 'unnaturalness' of these drugs makes some people uneasy in a way that caffeine does not. The claim, repeated in many responses to our survey is that using such drugs, or any performance-enhancing drug, makes accomplishments somehow less worthy because they aren't natural. But again, what is 'natural'? Devices such as glasses, hearing aids, pacemakers and artificial hips are unnatural. Yet they are widely accepted as legitimate ways to enhance the human experience. By the same token, if drugs enhance performance on a standardized test, what is so 'natural' about prep courses designed to improve scores?

Ultimately, our visceral concept of what is 'natural' depends on what we are used to, and will continue to evolve as technology does. But in the meantime, we should not allow it to distract us from the rational consideration of deeper and more important ethical issues. In the case of Beatie and his wife, the elemental questions are the health, safety and emotional security of the child. Trying to decide such issues simply by fixating on a fluid and arbitrary definition is, by nature, silly.

\section{Ready or not}

\section{Transparency and honesty are essential if the genetic-testing industry is to live up to its potential.}

$\mathrm{N}$ avigenics, a California start-up company with solid backing, launched its flagship product this week. The Health Compass, a US $\$ 2,500$ genetic test, is being offered to consumers directly, over the Internet. It will scan DNA from a customer's saliva sample for a host of tiny variations and pronounce on the person's risk of developing 18 common medical conditions, including heart attack, prostate cancer and type 2 diabetes.

Why now? Because, as the company says in its corporate literature, "the science is ready. Genetic testing is ready to enter into common health-care practice."

Certainly genetic testing is here, ready or not. In the past year, genome-wide association studies have begun to pour out of labs, linking the blips in our genetic make-up to risks of developing particular medical conditions. Whether people would or could change their behaviour to ameliorate these risks remains unclear. But the ink on the research papers is barely dry before companies unveil commercial versions of the tests. Navigenics is simply the most recent; others include high-profile players such as 23 andMe in Mountain View California (see Nature 450, 11; 2007) and the Icelandic genomics company deCODE Genetics. They also encompass smaller operations such as Philadelphia-based Smart Genetics, which last month offered a genetic assessment of the risk of Alzheimer's disease. Rarely have basic discoveries morphed into a commercial product quite so swiftly.

Following almost as quickly are the concerns being raised about the use of such tests. Many of these worries have been around for some time, but now that the tests are here, these discussions have taken on a new urgency. If consumers are to reap the benefits that genetic testing can offer, they need understandable information about the basis, validity and limitations of the tests. One proposed structure for providing this information is a publicly accessible registry into which test-makers would be required to upload data about their tests and the studies that back them. This information should be updated as genetic risks are changed or refined, as inevitably they will be.

Such a registry should be international, harmonizing information in what will doubtless be an industry without borders. This approach seems preferable to stepped-up regulation by agencies such as the Food and Drug Administration (FDA), which - in addition to travelling at the snail's pace of bureaucracy rather than the lightning speed of burgeoning markets - could easily have the effect of driving lessthan-desirable players underground, where sub-standard tests will remain as easy to buy as black-market DVDs.

Many critics would say that a hands-off approach by the FDA is irresponsible. But on what basis should genetic tests be treated any differently from others done in government-certified clinical labs? Many of these tests - including almost all of those done by labs inhouse, rather than sent out to patients and doctors as kits - are not required to demonstrate clinical utility on a test-by-test basis. For that matter, why should genetic tests be treated differently from medical devices such as MRI scanners, which were left by the FDA to prove their utility and clinical validity to physicians and providers in the marketplace, rather than in pre-market assessments?

It would be naive to suggest that transparency will solve all problems, or to assume that the marketplace will separate the wheat from the chaff with unfailing accuracy and efficiency. But to advocate relatively light regulation does not mean turning a blind eye to the risks of such a strategy. It means taking seriously the presumption that people should be free to inform themselves and make their own choices, and that by doing so they may benefit not just themselves but also the overall pace of innovation. Should it become clear that the system is allowing harm, then enhanced regulation will be appropriate.

It is also worth noting that the scientists who have driven this revolution need to assume a prominent role in ensuring that its benefits are not mishandled. Those who start companies, or advise them, can and must lead the way in ensuring that their enterprises are transparent and valid. In the meantime, online shoppers who buy genetic tests would do well to keep asking themselves whether the science is, indeed, ready. 